\title{
Single and Double Suzuki-Miyaura Couplings with Symmetric Dihalobenzenes
}

\author{
David J. Sinclair and Michael S. Sherburn*
}

Research School of Chemistry, Australian National University, Canberra, ACT 0200, Australia

\author{
sherburn@rsc.anu.edu.au
}

\begin{abstract}
Contents
Page

Characterization for compounds 4, 5-meta, 5-para, 6-meta, 6-para, 7, S1-S4, 8, S5-S8, 9, S9-S14, 10, 11, S15, 12, S16, 13, S17, and 14-18. S2-S13

${ }^{1} \mathrm{H}$ and ${ }^{13} \mathrm{C}$ NMR spectra of compounds 4, 5-meta, 5-para, S1, S5, S13, and 14-18. $\quad$ S14-S35
\end{abstract}




\section{Characterization Data}

Pinacolyl 2,6-dimethoxyphenylboronate (4)

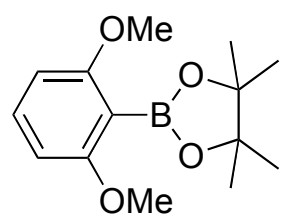

4

Mp 82-84 ${ }^{\circ} \mathrm{C}$ (hexanes); IR (film) 2977, 2936, 2836, 1599, 1584, 1466, 1430, 1378, 1344, 1307, 1244 , 1146, 1107, 1058, 859, 777, 725, $678 \mathrm{~cm}^{-1} ;{ }^{1} \mathrm{H}$ NMR $\left(300 \mathrm{MHz}, \mathrm{CDCl}_{3}\right) \delta 7.23(\mathrm{t}, J=8.3 \mathrm{~Hz}, 1 \mathrm{H})$, $6.46(\mathrm{~d}, J=8.4 \mathrm{~Hz}, 2 \mathrm{H}), 3.76(\mathrm{~s}, 6 \mathrm{H}), 1.38(\mathrm{~s}, 12 \mathrm{H}) ;{ }^{13} \mathrm{C} \mathrm{NMR}\left(75 \mathrm{MHz}, \mathrm{CDCl}_{3}\right) \delta 163.4,131.4$, 103.3, 83.7, 55.7, 24.7; MS (EI) $m / z 264\left(\mathrm{M}^{+}\right)$; HRMS (EI) $m / z$ calcd for $\mathrm{C}_{14} \mathrm{H}_{21} \mathrm{BO}_{4}\left(\mathrm{M}^{+}\right)$264.1533, found 264.1532 .

2,6-Dimethoxy-3'-iodobiphenyl (5-meta)

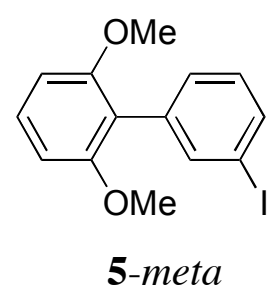

Mp 85-86 ${ }^{\circ} \mathrm{C}$ (DCM/ethanol); IR (film) 2935, 2835, 1589, 1471, 1432, 1244, 1110, 788, 729, 695 $\mathrm{cm}^{-1} ;{ }^{1} \mathrm{H}$ NMR $\left(300 \mathrm{MHz}, \mathrm{CDCl}_{3}\right) \delta 7.71(\mathrm{t}, J=1.7 \mathrm{~Hz}, 1 \mathrm{H}), 7.65(\mathrm{ddd}, J=7.8,1.7,1.1 \mathrm{~Hz}, 1 \mathrm{H})$, 7.35-7.27 (m, $2 \mathrm{H}), 7.15(\mathrm{t}, J=7.8 \mathrm{~Hz}, 1 \mathrm{H}), 6.65(\mathrm{~d}, J=8.1 \mathrm{~Hz}, 2 \mathrm{H}), 3.75(\mathrm{~s}, 6 \mathrm{H}) ;{ }^{13} \mathrm{C}$ NMR $(75$ $\left.\mathrm{MHz}, \mathrm{CDCl}_{3}\right) \delta 157.5,139.7,136.4,135.6,130.3,129.4,129.2,117.9,104.1,93.7,55.9 ; \mathrm{MS}(\mathrm{EI}) \mathrm{m} / \mathrm{z}$ $340\left(\mathrm{M}^{+}\right)$; HRMS (EI) $m / z$ calcd for $\mathrm{C}_{14} \mathrm{H}_{13} \mathrm{IO}_{2}\left(\mathrm{M}^{+}\right)$339.9960, found 339.9967 . 


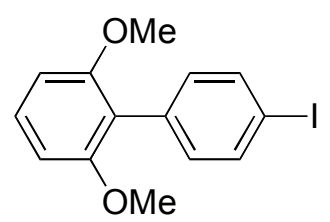

5-para

Mp 129-130 ${ }^{\circ} \mathrm{C}$ (DCM/ethanol); IR (film) 2988, 2937, 2833, 1588, 1469, 1431, 1249, 1110, 999, 820, 779, 729, $701 \mathrm{~cm}^{-1} ;{ }^{1} \mathrm{H}$ NMR $\left(300 \mathrm{MHz}, \mathrm{CDCl}_{3}\right) \delta 7.73(\mathrm{~d}, J=8.4 \mathrm{~Hz}, 2 \mathrm{H}), 7.29(\mathrm{t}, J=8.3 \mathrm{~Hz}, 1 \mathrm{H})$, $7.11(\mathrm{~d}, J=8.4 \mathrm{~Hz}, 2 \mathrm{H}), 6.65(\mathrm{~d}, J=8.1 \mathrm{~Hz}, 2 \mathrm{H}), 3.74(\mathrm{~s}, 6 \mathrm{H}) ;{ }^{13} \mathrm{C} \mathrm{NMR}\left(75 \mathrm{MHz}, \mathrm{CDCl}_{3}\right) \delta 157.5$, 136.8, 133.7, 133.0, 129.0, 118.3, 104.2, 92.6, 55.9; MS (EI) $m / z 340\left(\mathrm{M}^{+}\right)$; Anal. Calcd for $\mathrm{C}_{14} \mathrm{H}_{13} \mathrm{IO}_{2}$ : C 49.4\%, H 3.9. Found: C 49.7, H 4.0.

2,2",6,6"-Tetramethoxy-1,1':3',1"-terphenyl (6-meta)

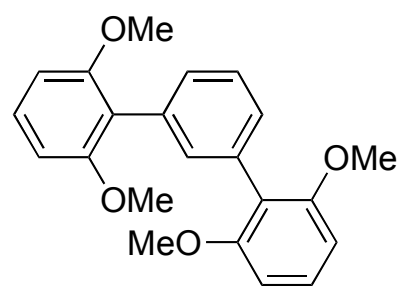

6-meta

Grewal, R. S.; Hart, H.; Vinod, T. K. J. Org. Chem. 1992, 57, 2721-2726.

2,2",6,6"-Tetramethoxy-1,1':4',1"-terphenyl (6-para)

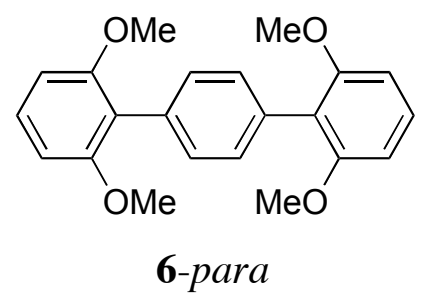

Kern, W.; Ebersbach, H. W.; Ziegler, I. Makromolekulare Chemie 1959, 31, 154-180. 
Pinacolyl 4-methoxyphenylboronate (7)

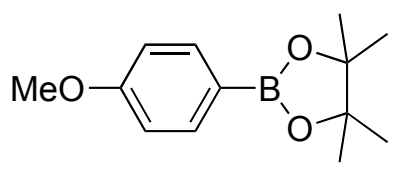

7

Ishiyama, T.; Murata, M.; Miyaura, N. J. Org. Chem. 1995, 60, 7508-7510.

3-Iodo-4'-methoxybiphenyl (S1; Table 2, entry 3, monocoupled product)

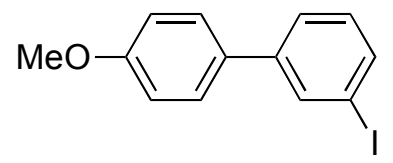

S1

Mp 111-112 ${ }^{\circ} \mathrm{C}$ (DCM/ethanol); IR (film) 2959, 2931, 2836, 1607, 1554, 1518, 1251, 1037, 1018, 838, 781, $684 \mathrm{~cm}^{-1} ;{ }^{1} \mathrm{H}$ NMR $\left(300 \mathrm{MHz}, \mathrm{CDCl}_{3}\right) \delta 7.91(\mathrm{t}, J=1.7 \mathrm{~Hz}, 1 \mathrm{H}), 7.63(\mathrm{ddd}, J=8.0,1.7,1.2$ $\mathrm{Hz}, 1 \mathrm{H}), 7.52-7.46(\mathrm{~m}, 3 \mathrm{H}), 7.14(\mathrm{t}, J=7.8 \mathrm{~Hz}, 1 \mathrm{H}), 6.98(\mathrm{~d}, J=9.0 \mathrm{~Hz}, 2 \mathrm{H}), 3.85(\mathrm{~s}, 3 \mathrm{H}) ;{ }^{13} \mathrm{C}$ NMR (75 MHz, $\left.\mathrm{CDCl}_{3}\right) \delta$ 159.6, 143.1, 135.7, 135.5, 132.1, 130.4, 128.2, 126.0, 114.3, 94.8, 55.4; MS (EI) $m / z 310\left(\mathrm{M}^{+}\right)$; Anal. Calcd for $\mathrm{C}_{13} \mathrm{H}_{11} \mathrm{IO}$ : C 50.4\%, H 3.6. Found: C 50.2, H 3.8.

4-Iodo-4'-methoxybiphenyl (S2; Table 2, entry 4, monocoupled product)

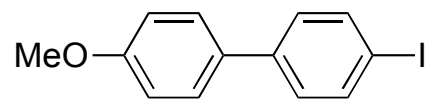

S2

Spivey, A. C.; Diaper, C. M.; Adams, H.; Rudge, A. J. J. Org. Chem. 2000, 65, 5253-5263. 
4,4"-Dimethoxy-1,1':3',1"-terphenyl (S3; Table 2, entry 3, biscoupled product)

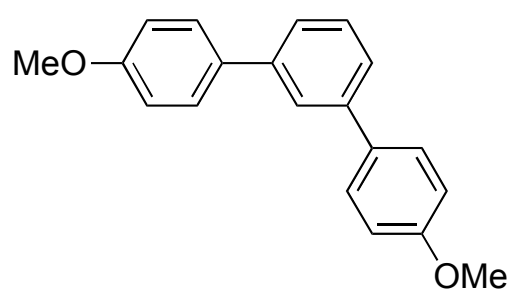

S3

* Commercially available

Mueller, G. P.; Honaker, C. B. J. Am. Chem. Soc. 1951, 73, 2377-2379.

4,4"-Dimethoxy-1,1':4',1"-terphenyl (S4; Table 2, entry 4, biscoupled product)

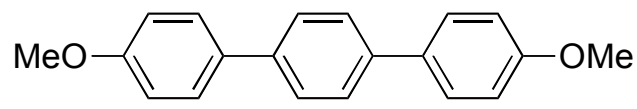

\section{S4}

Tao, X.; Zhao, Y.; Shen, D. Synlett 2002, 757-760.

Pinacolyl 4-nitrophenylboronate (8)

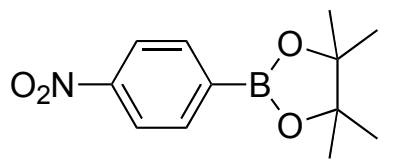

8

* Commercially available

3-Iodo-4'-nitrobiphenyl (S5; Table 2, entry 5, monocoupled product)

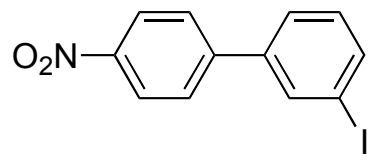

S5 
Mp 125-127 ${ }^{\circ} \mathrm{C}$ (DCM/ethanol); IR (film) 2917, 2849, 1597, 1557, 1513, 1468, 1344, 1108, 854, 782, 768, 748, $687 \mathrm{~cm}^{-1} ;{ }^{1} \mathrm{H}$ NMR $\left(300 \mathrm{MHz}, \mathrm{CDCl}_{3}\right) \delta 8.30(\mathrm{~d}, J=9.0 \mathrm{~Hz}, 2 \mathrm{H}), 7.96(\mathrm{t}, J=1.8 \mathrm{~Hz}, 1 \mathrm{H})$, $7.78(\mathrm{dt}, J=8.1,1.4 \mathrm{~Hz}, 1 \mathrm{H}), 7.70(\mathrm{~d}, J=9.0 \mathrm{~Hz}, 2 \mathrm{H}), 7.58(\mathrm{ddd}, J=7.8,1.8,0.9 \mathrm{~Hz}, 1 \mathrm{H}), 7.23(\mathrm{t}, J$ $=8.0 \mathrm{~Hz}, 1 \mathrm{H}) ;{ }^{13} \mathrm{C} \mathrm{NMR}\left(75 \mathrm{MHz}, \mathrm{CDCl}_{3}\right) \delta 147.4,145.9,140.9,137.8,136.3,130.8,127.9,126.6$, 124.2, 95.0; MS (EI) $m / z 325\left(\mathrm{M}^{+}\right)$; HRMS (EI) $\mathrm{m} / z$ calcd for $\mathrm{C}_{12} \mathrm{H}_{8} \mathrm{INO}_{2}\left(\mathrm{M}^{+}\right)$324.9600, found 324.9608 .

4-Iodo-4'-nitrobiphenyl (S6; Table 2, entry 6, monocoupled product)

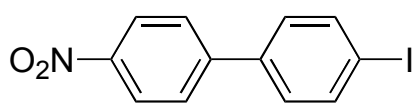

S6

* Commercially available

1) Sarma, J. A. R. P.; Allen, F. H.; Hoy, V. J.; Howard, J. A. K.; Thaimattam, R.; Kumar, B.; Desiraju, G. R. Chem. Commun. 1997, 101-102. 2) Harley-Mason, J.; Mann, F. G. J. Chem. Soc. 1940, $1374-1385$.

4,4"-Dinitro-1,1':3',1"-terphenyl (S7; Table 2, entry 5, biscoupled product)

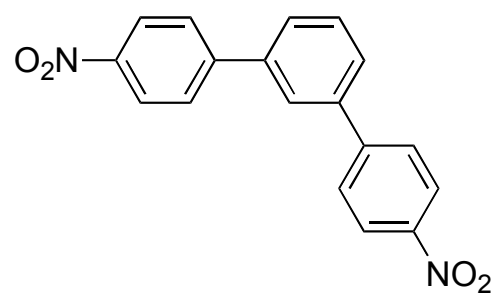

S7

Descotes, G.; Praly, J. P. Bull. Soc. Chim. Fr. 1976, 901-910. 
4,4"-Dinitro-1,1':4',1"-terphenyl (S8; Table 2, entry 6, biscoupled product)

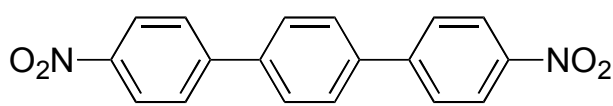

S8

* Commercially available

Chaumeil, H.; Le Drian, C.; Defoin, A. Synthesis 2002, 757-760.

Pinacolyl phenylboronate (9)

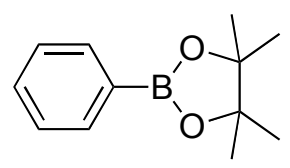

9

* Commercially available

Ishiyama, T.; Murata, M.; Miyaura, N. J. Org. Chem. 1995, 60, 7508-7510.

3-Iodobiphenyl (S9; Table 2, entry 7, monocoupled product)

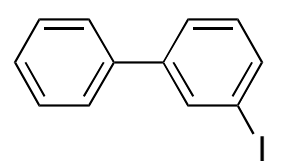

S9

Dektar, J. H.; Hacker, N. P. J. Org. Chem. 1990, 55, 639-647.

4-Iodobiphenyl (S10; Table 2, entry 8, monocoupled product)

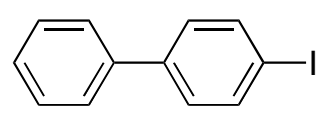

S10

* Commercially available

Dektar, J. H.; Hacker, N. P. J. Org. Chem. 1990, 55, 639-647. 
1,1':3',1"-Terphenyl (S11; Table 2, entry 7, biscoupled product)

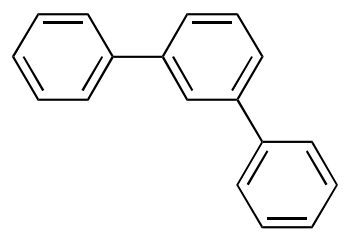

S11

* Commercially available

Asao, N.; Nogami, T.; Lee, S.; Yamamoto, Y. J. Am. Chem. Soc. 2003, 125, 10921-10925.

1,1':4',1"-Terphenyl (S12; Table 2, entry 8, biscoupled product)

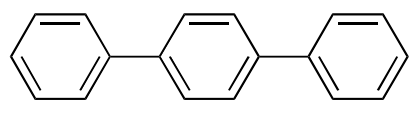

S12

* Commercially available

Voronkov, M. G.; Deryagina, E. N.; Sukhomazova, E. N.; Klochkova, L. G. Zhurnal Organicheskoi Khimii 1975, 11, 1131.

3-Bromo-2',6'-dimethoxybiphenyl (S13; Table 3, entry 4, monocoupled product)

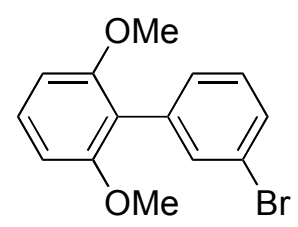

S13

Mp 79-80 C (DCM/ethanol); IR (film) 2937, 2835, 1589, 1472, 1242, 1111, 790, 729, 694, $658 \mathrm{~cm}^{-1}$; ${ }^{1} \mathrm{H}$ NMR $\left(300 \mathrm{MHz}, \mathrm{CDCl}_{3}\right) \delta 7.52(\mathrm{dd}, J=2.1,1.2 \mathrm{~Hz}, 1 \mathrm{H}), 7.45(\mathrm{ddd}, J=6.0,3.0,2.0 \mathrm{~Hz}, 1 \mathrm{H})$, $7.33-7.28(\mathrm{~m}, 3 \mathrm{H}), 6.66(\mathrm{~d}, J=8.4 \mathrm{~Hz}, 2 \mathrm{H}), 3.75(\mathrm{~s}, 6 \mathrm{H}) ;{ }^{13} \mathrm{C} \mathrm{NMR}\left(75 \mathrm{MHz}, \mathrm{CDCl}_{3}\right) \delta 157.5$, 
136.3, 133.9, 129.7, 129.7, 129.2, 129.1, 121.7, 118.0, 104.1, 55.9; MS (EI) m/z 292/294 (M+); Anal. Calcd for $\mathrm{C}_{14} \mathrm{H}_{13} \mathrm{BrO}_{2}$ : C 57.4\%, H 4.5. Found: C 57.3, $\mathrm{H} 4.4$.

4-Bromo-2',6'-dimethoxybiphenyl (S14; Table 3, entry 5, monocoupled product)

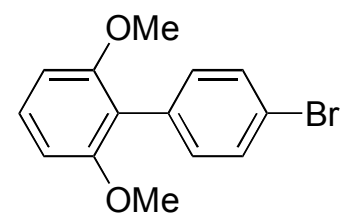

S14

von dem Bussche-Huennefeld, C.; Helgeson, R. C.; Buerhing, D.; Knobler, C. B.; Cram, D. J. Croat. Chim. Acta 1996, 69, 447-458.

2,6-Dimethoxyphenylboronic acid (10)

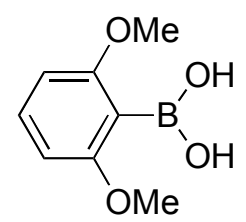

10

* Commercially available

von dem Bussche-Huennefeld, C.; Helgeson, R. C.; Buerhing, D.; Knobler, C. B.; Cram, D. J. Croat. Chim. Acta 1996, 69, 447-458.

4-Methoxyphenylboronic acid (11)

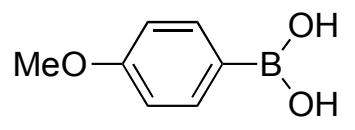

11

* Commercially available 
4-Bromo-4'-methoxybiphenyl (S15; Table 3, entry 10, monocoupled product)

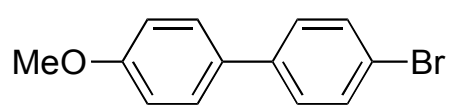

S15

* Commercially available

Spivey, A. C.; Diaper, C. M.; Adams, H.; Rudge, A. J. J. Org. Chem. 2000, 65, 5253-5263.

4-Nitrophenylboronic acid (12)

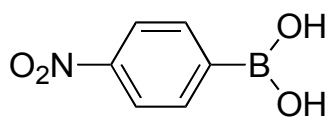

12

* Commercially available

4-Bromo-4'-nitrobiphenyl (S16; Table 3, entry 12, monocoupled product)

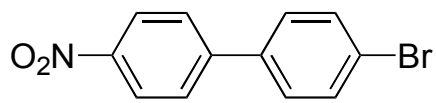

\section{S16}

* Commercially available

Kim, Y.; Han, K.; Ha, C.-S. Macromolecules 2002, 35, 8759-8767.

Phenylboronic acid (13)

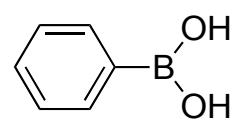

13

* Commercially available 
4-Bromobiphenyl (S17; Table 3, entry 13, monocoupled product)

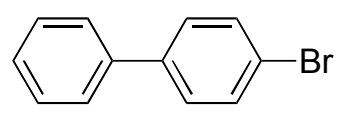

S17

* Commercially available

Berthiol, F.; Kondolff, I.; Doucet, H.; Santelli, M. J. Organomet. Chem. 2004, 689, 2786-2798.

2,6-Dimethoxy-4"-iodo-1,1':4',1"-terphenyl (14)

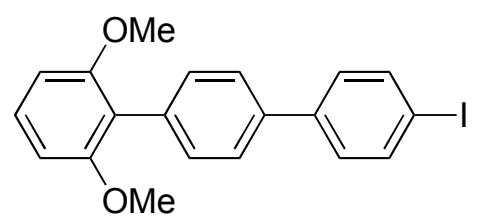

14

Mp 157-158 ${ }^{\circ} \mathrm{C}$ (DCM/ethanol); IR (film) 2932, 2835, 1588, 1470, 1245, 1105, 1001, 814, $735 \mathrm{~cm}^{-1}$; ${ }^{1} \mathrm{H}$ NMR $\left(300 \mathrm{MHz}, \mathrm{CDCl}_{3}\right) \delta 7.77(\mathrm{~d}, J=8.4 \mathrm{~Hz}, 2 \mathrm{H}), 7.60(\mathrm{~d}, J=8.4 \mathrm{~Hz}, 2 \mathrm{H}), 7.45(\mathrm{~d}, J=8.4 \mathrm{~Hz}$, $2 \mathrm{H}), 7.40(\mathrm{~d}, J=8.4 \mathrm{~Hz}, 2 \mathrm{H}), 7.31(\mathrm{t}, J=8.6 \mathrm{~Hz}, 1 \mathrm{H}), 6.69(\mathrm{~d}, J=8.4 \mathrm{~Hz}, 2 \mathrm{H}), 3.77(\mathrm{~s}, 6 \mathrm{H}) ;{ }^{13} \mathrm{C}$ NMR (75 MHz, $\left.\mathrm{CDCl}_{3}\right) \delta 157.7,140.8,138.2,137.8,133.7,131.5,129.0,128.8,126.2,118.8,104.2$, 92.77, 56.0; MS (EI) $m / z 416\left(\mathrm{M}^{+}\right)$; HRMS (EI) $m / z$ calcd for $\mathrm{C}_{20} \mathrm{H}_{17} \mathrm{IO}_{2}\left(\mathrm{M}^{+}\right)$416.0273, found 416.0274.

2,2"',6,6"'-Tetramethoxy-1,1':4',1":4",1'"-quaterphenyl (15)

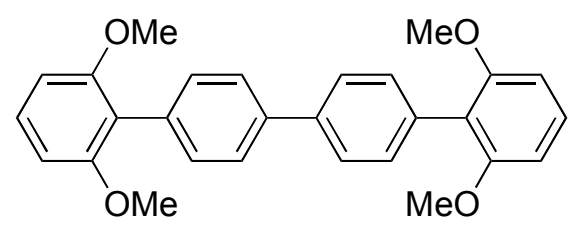

15

Mp $285^{\circ} \mathrm{C}$ (DCM/ethanol); IR (film) 2989, 2936, 2835, 1586, 1468, 1431, 1247, 1110, 821, 782, 732 $\mathrm{cm}^{-1} ;{ }^{1} \mathrm{H}$ NMR $\left(300 \mathrm{MHz}, \mathrm{CDCl}_{3}\right) \delta 7.69(\mathrm{~d}, J=8.1 \mathrm{~Hz}, 4 \mathrm{H}), 7.45(\mathrm{~d}, J=8.4 \mathrm{~Hz}, 4 \mathrm{H}), 7.30(\mathrm{t}, J=8.4$ 
$\mathrm{Hz}, 2 \mathrm{H}), 6.69(\mathrm{~d}, J=8.4 \mathrm{~Hz}, 4 \mathrm{H}), 3.78(\mathrm{~s}, 12 \mathrm{H}) ;{ }^{13} \mathrm{C} \mathrm{NMR}\left(75 \mathrm{MHz}, \mathrm{CDCl}_{3}\right) \delta 157.8,139.6,132.8$, 131.2, 128.7, 126.5, 119.2, 104.3, 56.0; MS (EI) $m / z 426\left(\mathrm{M}^{+}\right)$; Anal. Calcd for $\mathrm{C}_{28} \mathrm{H}_{26} \mathrm{O}_{4}: \mathrm{C} 78.9 \%$, $\mathrm{H}$ 6.1. Found: C 78.7, H 6.4.

3,5-Diiodo-2',6'-dimethoxybiphenyl (16)<smiles>COc1cccc(OC)c1-c1cc(I)cc(I)c1</smiles>

16

Mp 170-172 ${ }^{\circ} \mathrm{C}$ (DCM/ethanol); IR (film) 2917, 2835, 1590, 1532, 1472, 1428, 1396, 1249, 1236, 1108, 846, 778, $700 \mathrm{~cm}^{-1} ;{ }^{1} \mathrm{H}$ NMR $\left(300 \mathrm{MHz}, \mathrm{CDCl}_{3}\right) \delta 7.98(\mathrm{t}, J=1.7 \mathrm{~Hz}, 1 \mathrm{H}), 7.64(\mathrm{~d}, J=1.5 \mathrm{~Hz}$, $2 \mathrm{H}), 7.29(\mathrm{t}, J=8.6 \mathrm{~Hz}, 1 \mathrm{H}), 6.62(\mathrm{~d}, J=8.7 \mathrm{~Hz}, 2 \mathrm{H}), 3.75(\mathrm{~s}, 6 \mathrm{H}) ;{ }^{13} \mathrm{C} \mathrm{NMR}\left(75 \mathrm{MHz}, \mathrm{CDCl}_{3}\right)$ $\delta$ 157.4, 143.1, 139.2, 138.0, 129.7, 116.4, 104.0, 93.9, 55.9; MS (EI) $\mathrm{m} / z 466\left(\mathrm{M}^{+}\right)$; HRMS (EI) $\mathrm{m} / z$ calcd for $\mathrm{C}_{14} \mathrm{H}_{12} \mathrm{I}_{2} \mathrm{O}_{2}\left(\mathrm{M}^{+}\right)$465.8927, found 465.8936.

5'-Iodo-2,2",6,6"-tetramethoxy-1,1':3',1"-terphenyl (17)

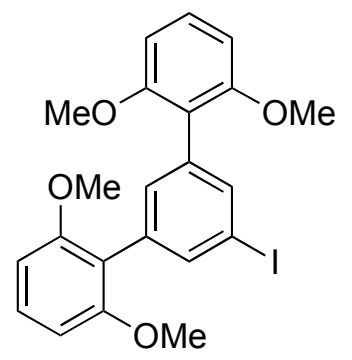

17

Mp 220-222 ${ }^{\circ} \mathrm{C}$ (DCM/ethanol); IR (film) 2936, 2837, 1589, 1472, 1433, 1416, 1249, 1112, 784, 731, $702 \mathrm{~cm}^{-1} ;{ }^{1} \mathrm{H}$ NMR $\left(300 \mathrm{MHz}, \mathrm{CDCl}_{3}\right) \delta 7.65(\mathrm{~d}, J=1.2 \mathrm{~Hz}, 2 \mathrm{H}), 7.35(\mathrm{t}, J=1.2 \mathrm{~Hz}, 1 \mathrm{H}), 7.27(\mathrm{t}, J=$ $8.6 \mathrm{~Hz}, 2 \mathrm{H}), 6.64(\mathrm{~d}, J=8.4 \mathrm{~Hz}, 4 \mathrm{H}), 3.75(\mathrm{~s}, 12 \mathrm{H}) ;{ }^{13} \mathrm{C} \mathrm{NMR}\left(75 \mathrm{MHz}, \mathrm{CDCl}_{3}\right) \delta 157.7,138.0$, 
134.9, 133.4, 128.9, 118.3, 104.2, 92.7, 55.9; MS (EI) $m / z 476\left(\mathrm{M}^{+}\right)$; HRMS (EI) $m / z$ calcd for $\mathrm{C}_{22} \mathrm{H}_{21} \mathrm{IO}_{4}\left(\mathrm{M}^{+}\right)$476.0485, found 476.0485.

5'-(2,6-dimethoxyphenyl)-2,2",6,6"-tetramethoxy-1,1':3',1"-terphenyl (18)

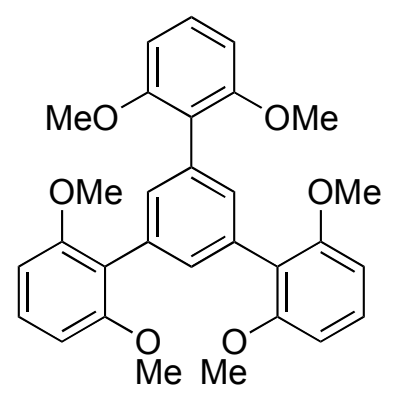

18

Mp 253-254 ${ }^{\circ} \mathrm{C}$ (DCM/ethanol); IR (film) 2936, 2836, 1599, 1586, 1471, 1431, 1414, 1248, 1236 , 1111, 779, 729, $707 \mathrm{~cm}^{-1} ;{ }^{1} \mathrm{H}$ NMR (300 MHz, $\left.\mathrm{CDCl}_{3}\right) \delta 7.41(\mathrm{~s}, 3 \mathrm{H}), 7.24(\mathrm{t}, J=8.3 \mathrm{~Hz}, 3 \mathrm{H}), 6.66$ $(\mathrm{d}, J=8.4 \mathrm{~Hz}, 6 \mathrm{H}), 3.73(\mathrm{~s}, 18 \mathrm{H}) ;{ }^{13} \mathrm{C} \mathrm{NMR}\left(75 \mathrm{MHz}, \mathrm{CDCl}_{3}\right) \delta$ 158.0, 132.5, 131.4, 128.0, 120.4, 104.6, 56.0; MS (EI) $m / z 486\left(\mathrm{M}^{+}\right)$; HRMS (EI) $m / z$ calcd for $\mathrm{C}_{30} \mathrm{H}_{30} \mathrm{O}_{6}\left(\mathrm{M}^{+}\right)$486.2042, found 486.2047. 
STANDARD IH OBSERVE

Pulse Sequence: s2pu1

Solvent : $\mathrm{CDC} 13$

Temp. $25.0 \mathrm{C} / 298.1$

Pulse 90.0 degrees
Acq. time $3.744 \mathrm{sec}$

Width $4000.0 \mathrm{~Hz}$

OBSERVE H1, 299.9436006 MHZ

DATA PROCESSING

Total time $1 \mathrm{~min}, 0 \mathrm{sec}$

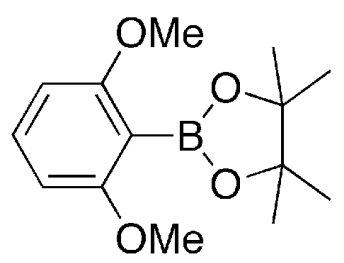

4

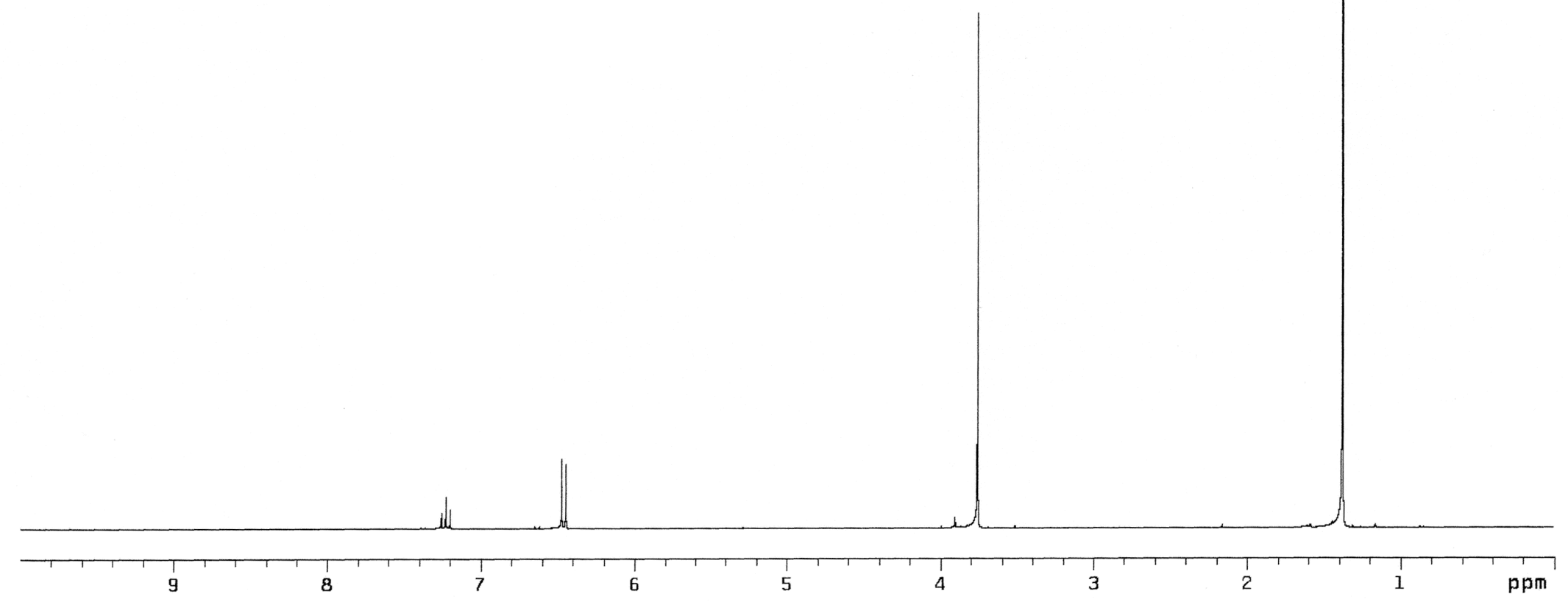


13C OBSERVE

Pulse Sequence: s2pul

Solvent: $\mathrm{CDC} 13$
Temp. $25.0 \mathrm{C} / 298.1 \mathrm{~K}$
INOVA-300 "purce11"

Relax. delay $1.000 \mathrm{sec}$

Acq. time 0.910 sec

288 ropetitions

OBSERE C13, $75.4208775 \mathrm{MHZ}$
DECOUPLE H1, 299.9450944 MHZ

Power $42 \mathrm{~dB}$

WALTZ-16 modulat

DATA PROCESSING $2.5 \mathrm{~Hz}$

Total time $5 \mathrm{hr}, 19 \mathrm{~min}, 49 \mathrm{sec}$

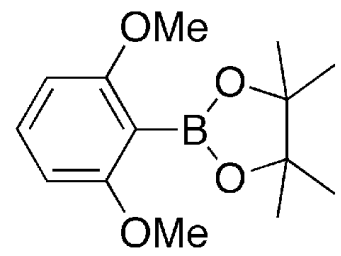

4

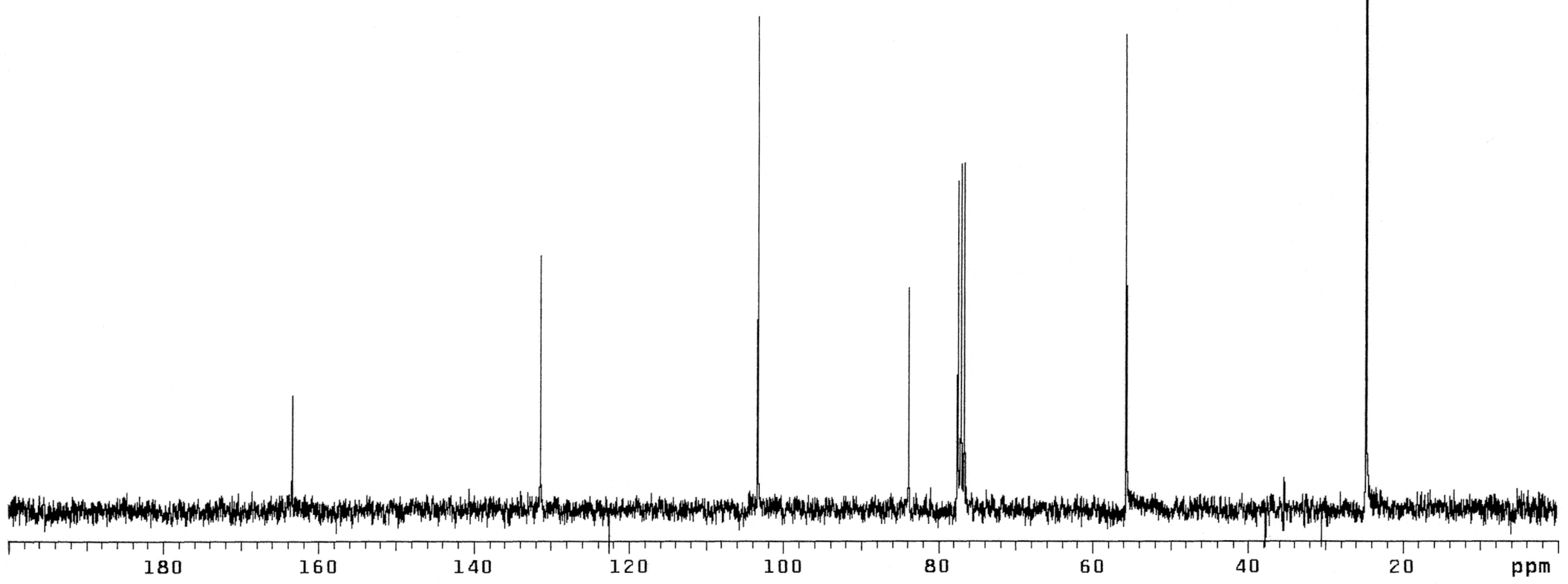


STANDARD IH OBSERVE

Pu1se Sequence: s2pu

Solvent: CDC13 $^{2}$

Tenp. $25.0 \mathrm{C} / 298.1 \mathrm{~K}$
INOYA-300 "purcel1"

Pulse 90.0 degrees

width $4000.0 \mathrm{HZ}$

16 repet Hions

DATA PROCESSING

FT size 32768
Total time $1 \mathrm{~min}, 0$ sec

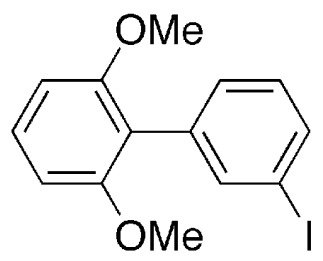

5-meta

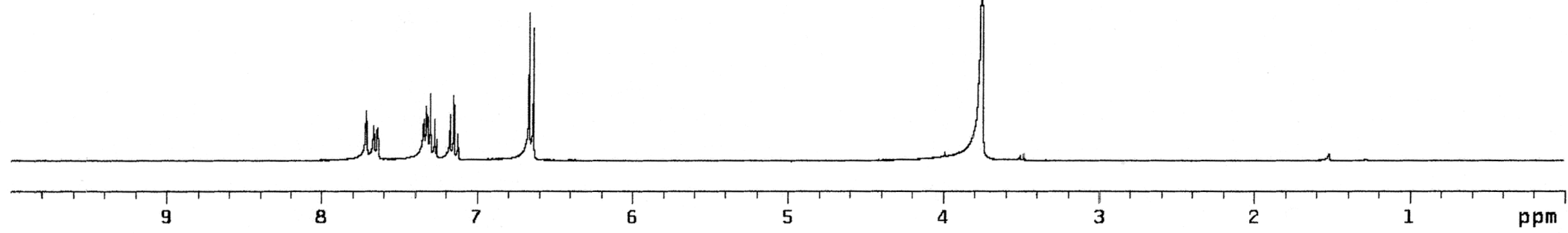


$13 \mathrm{C}$ OBSERVE

Pulse Sequence: s2pul

Solvent: $\mathrm{CDC} 13$

Temp. $25.0 \mathrm{C} / 298.1 \mathrm{~K}$
INOVA-300

Relax. delay $1.000 \mathrm{sec}$

Acq $t$ ime 0.910 sec

Width $18001.8 \mathrm{~Hz}$

704 repetitions
OBSERVE C13, 75.4

OECRVE $\mathrm{Cl}, \quad 75.4208786 \mathrm{MHZ}$

Power 42 dB

cont inuous ly on
WALT -16 modu

WALTZ-16 modulate
DATA PROCESSING

Line broadening $2.5 \mathrm{~Hz}$

Total time $5 \mathrm{hr}, 19 \mathrm{~min}, 49 \mathrm{sec}$

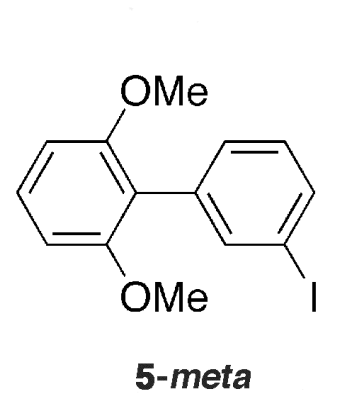

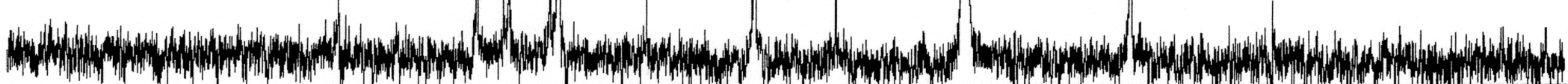
180

160 
STANDARD IH OBSERVE

Pulse Sequence: s2pur

Solvent: $\operatorname{CDC} 13$
Temp. $25.0 \mathrm{C} / 298.1 \mathrm{~K}$

Pulse 90.0 degrees

Acq. $t$ ime 3.744 sec

16 repetitions

OBSERVE H1, $299.9436006 \mathrm{MHZ}$

DATA PROCESSI
FT SIZE 32768

Total time $1 \mathrm{~min}, 0 \mathrm{sec}$

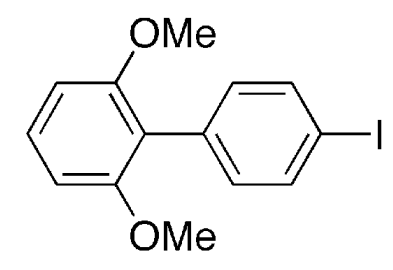

5-para

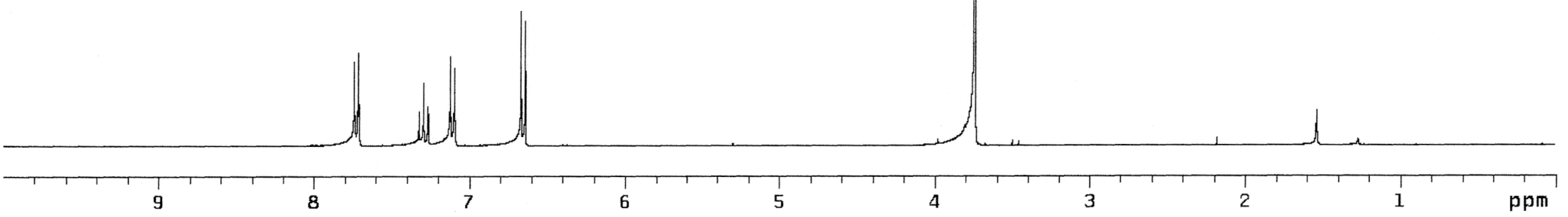




\section{C OBSERVE}

Pulse Sequence: s2pul

Solvent: $\mathrm{CDC} 13$

Temp. $25.0 \mathrm{C} / 298.1 \mathrm{~K}$
INOVA-300 "purce11"

Relax. delay $1.000 \mathrm{sec}$

Acq 45.0 degrees

Width $18001.8 \mathrm{~Hz}$

368 repetitions
OBSERVE C13, $75.4208775 \mathrm{MHZ}$
DECOUPL H1, 299.9450944 $\mathrm{MHZ}$

Power $42 \mathrm{~dB}$

continuous $1 y$ on

DATA PROCESSING

Line broadening $2.5 \mathrm{~Hz}$

Total time $5 \mathrm{hr}, 19 \mathrm{~min}, 49 \mathrm{sec}$

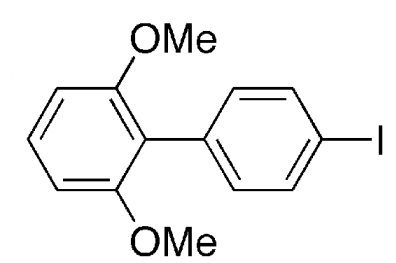

5-para

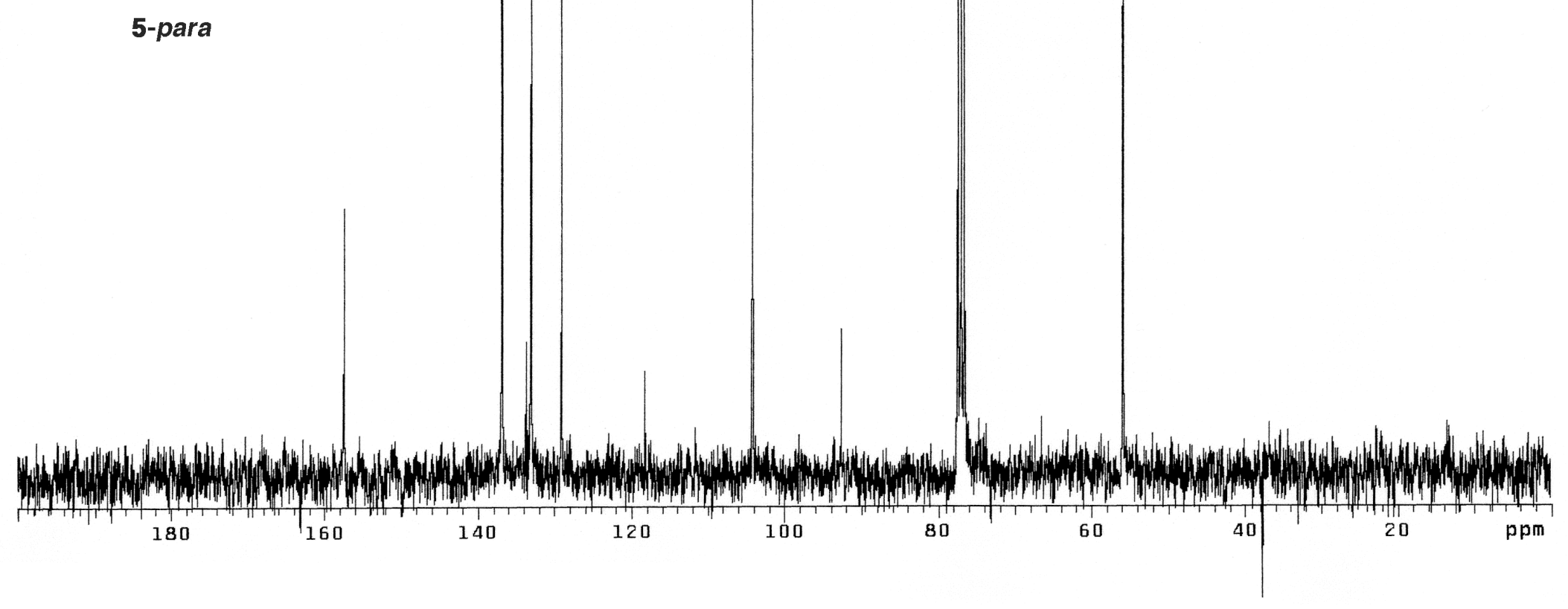


STANDARD IH OBSERVE

Pulse Sequence: s2pul

Solvent: $\mathrm{CDCl}$
Temp. $25.0 \mathrm{C} / 298.1 \mathrm{~K}$
INOVA-300 "purcel1"

Pulse 90.0 degrees

Acql time 3:.744 sec

16 repetitions

OBSERVE HI $299.9436006 \mathrm{MHZ}$

DATA PROCESS.ING

Total time $1 \mathrm{~min}, 0$ sec

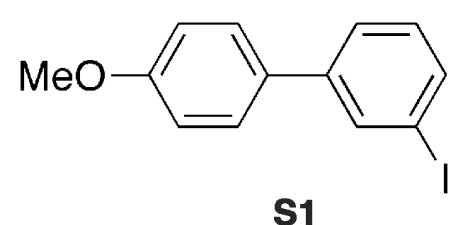

(a)

s

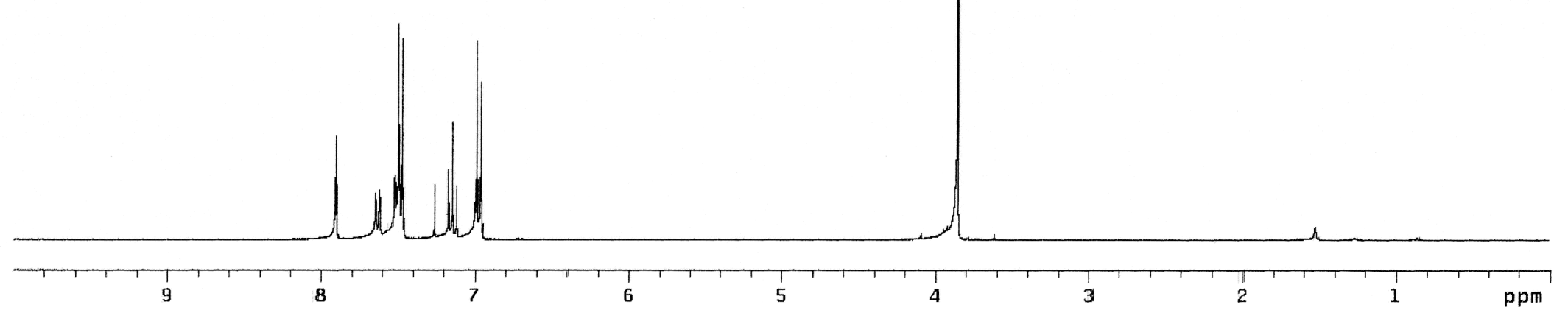


13C OBSERVE

Pulse Sequence: s2pur

Solvent: $\mathrm{CDCl}^{3}$

Temp. $25.0 \mathrm{C} / 298.1 \mathrm{~K}$
INOVA-300 "purcel1"

Relax delay $1.000 \mathrm{sec}$

Acq. time 0.910 sec

width $18001.8 \mathrm{~Hz}$

OBSERVE Cl3, $75.4208775 \mathrm{MHZ}$

POWer 42 dB

WALTZ-16 modulated

DATA PROCESSING

FT 512032768

rotal time $5 \mathrm{hr}, 19 \mathrm{~min}, 49 \mathrm{sec}$

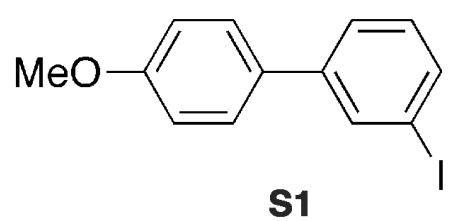

S1

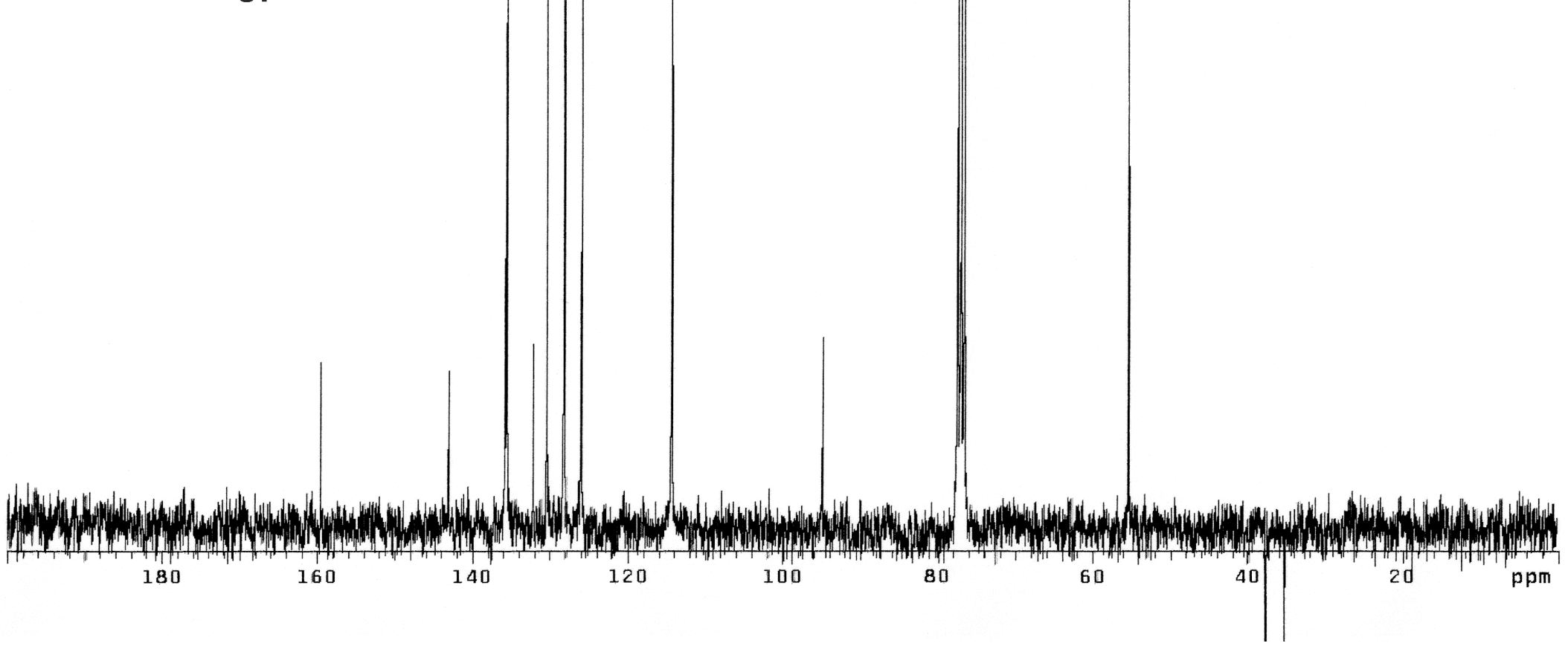


STANDARD IH OBSERVE

Pulse Sequence: s2put

Solvent: $\mathrm{CDCl}^{2}$,

Tenp. $25.0 \mathrm{C} / 298.1 \mathrm{~K}$
INOVA-300 "purcel1"

Pulse 90.0 degrees

Width $4000.0 \mathrm{~Hz}$

16 repetions

DATA PROCESSING

$\mathrm{FT}$ size 32768
Total time $1 \mathrm{~min}, 0 \mathrm{sec}$
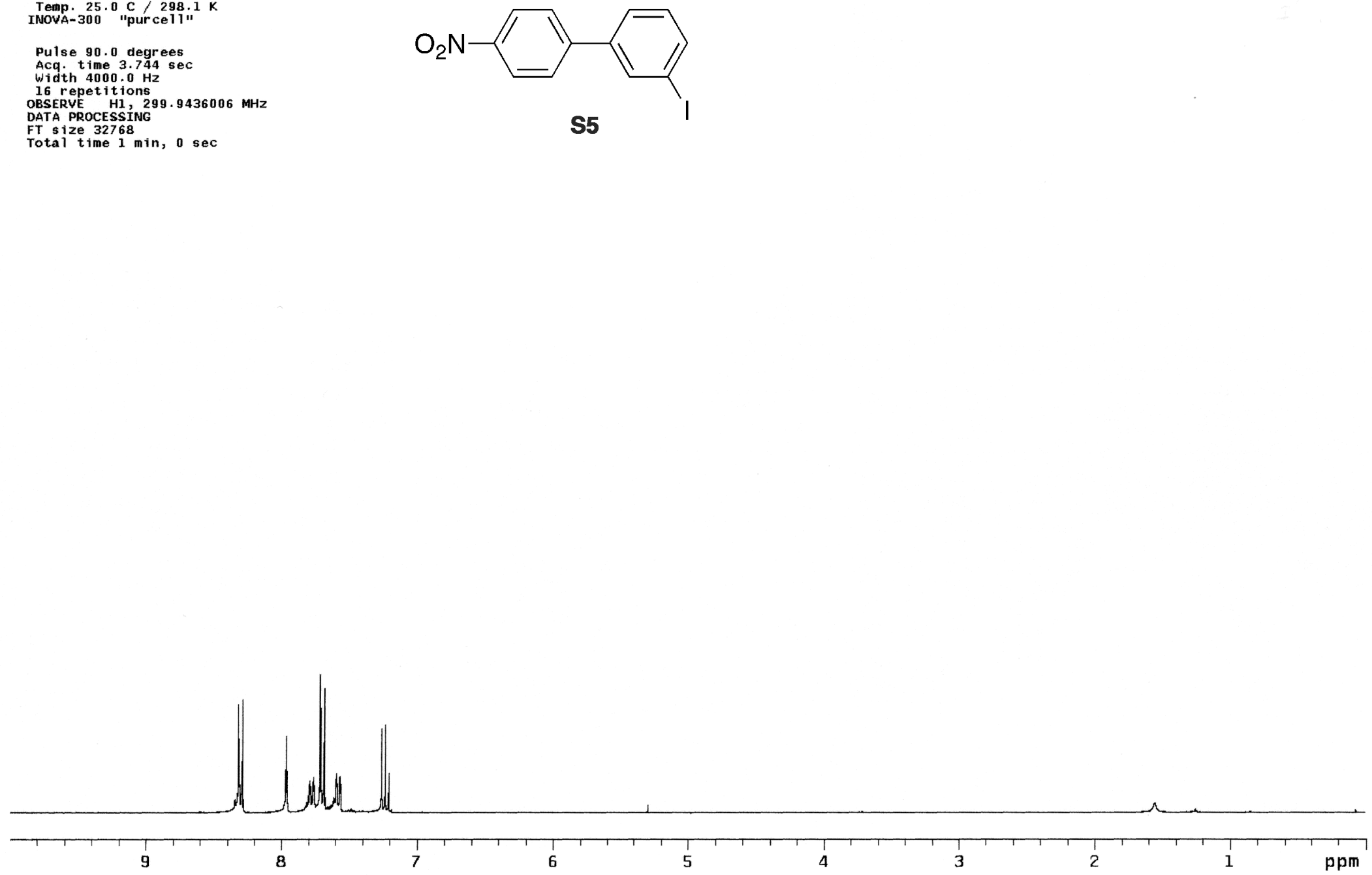
13C OBSERVE

Pulse Sequence: s2pul

Solvent $=\mathrm{CDC}^{13}$

Solvent: $\operatorname{CDC}^{2} 13298.1 \mathrm{~K}$
Temp. $25.0 \mathrm{C} / 298.1 \mathrm{~K}$
INOVA-300 "purce11"

Relax. delay $1.000 \mathrm{sec}$

Pulse 45.0 degrees
Acq. time 0.910 sec

Width $18001.8 \mathrm{~Hz}$

OBSERVE C13, $75.4208775 \mathrm{MHZ}$
DECOUPLE H1, 299.9450944 MHZ

Power 42 dB

Cont inuous ly on

DATA PROCESING $2.5 \mathrm{~Hz}$

FT size 32768
Total time $5 \mathrm{hr}, 19 \mathrm{~min}, 49 \mathrm{sec}$

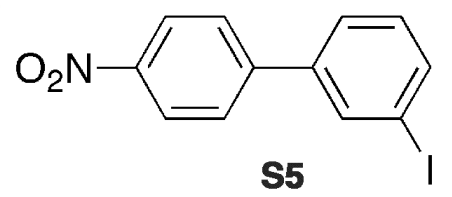

S5

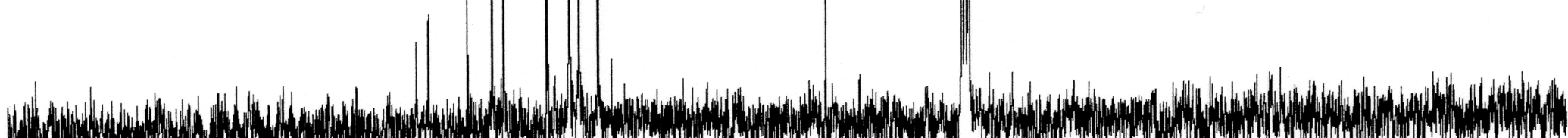

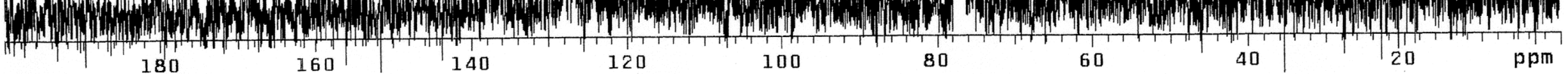


STANDARD IH OBSERVE

Pulse Sequence: s2pul

Solvent: $\operatorname{cDCl}^{3}$ 298.1

INOVA-300 "purcel1"

Pulse 90.0 degrees

Width $4000.0 \mathrm{~Hz}$

16 repet

DATA PROCESSING

$\mathrm{FT}$ size 32768
Total time $1 \mathrm{~min}, 0 \mathrm{sec}$
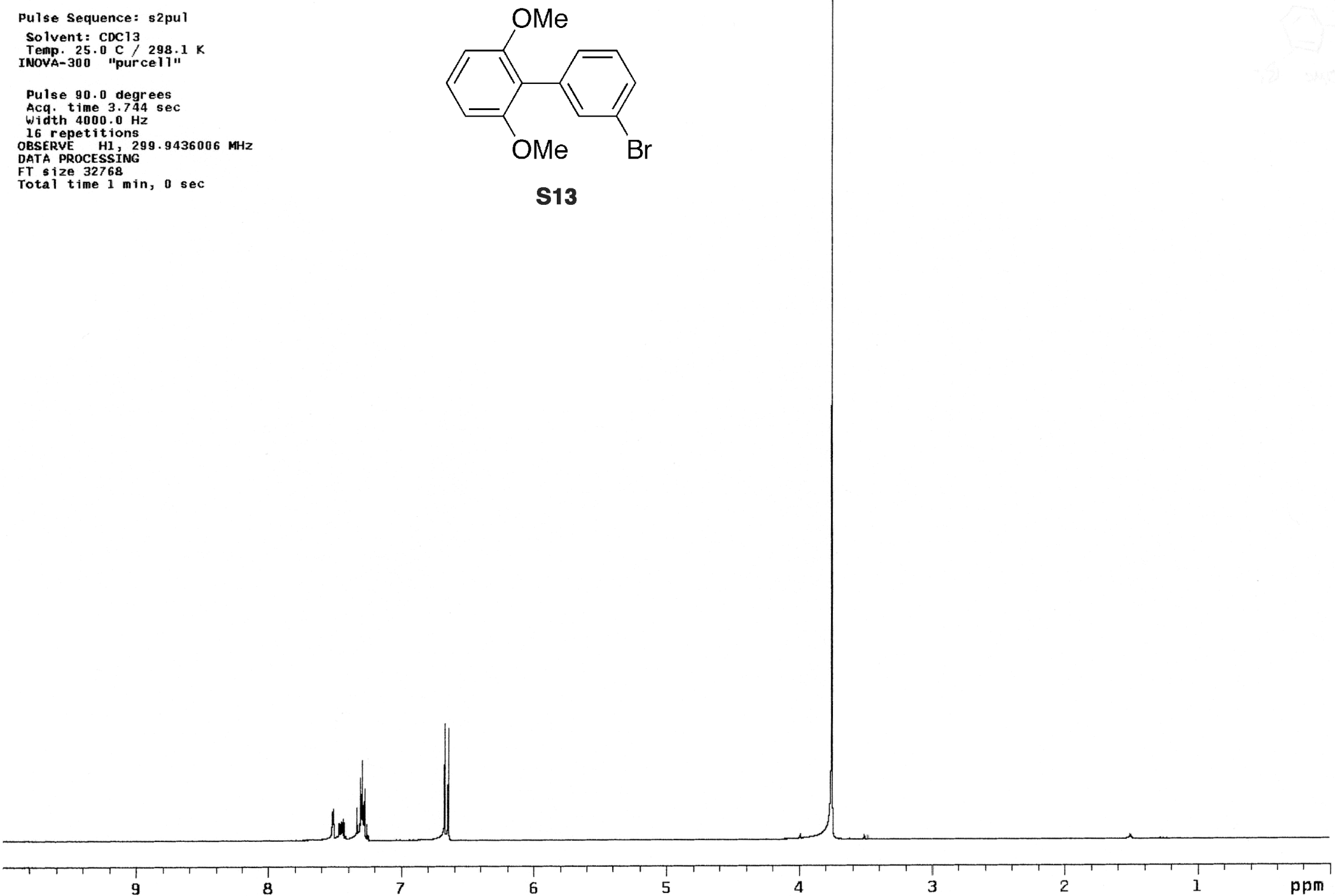
13C OBSERVE

Pulse Sequence: s2pul Solvent: $\mathrm{CDCl}$
Temp. $25.0 \mathrm{C} / 298.1 \mathrm{~K}$
INOVA-300 "purce11"

Relax. delay $1.000 \mathrm{sec}$

Acq. time $0.910 \mathrm{sec}$

Width $18001.8 \mathrm{~Hz}$

OBSERVE C13,75.4208786 MHZ

DECOUPLE HI, 299.9450944 MHZ

power 42 dB 20

WALTZ-16 moduTated

DATA PROCESSIAG

$2.5 \mathrm{~Hz}$

Total time $5 \mathrm{hr}, 19 \mathrm{~min}, 49 \mathrm{sec}$

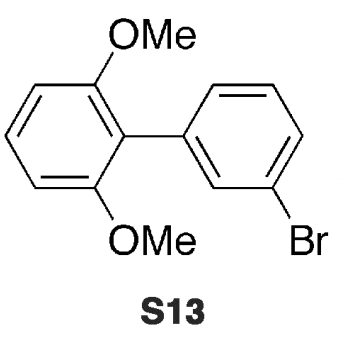

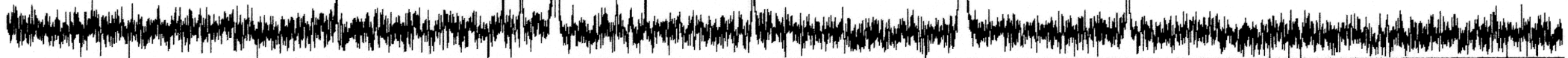

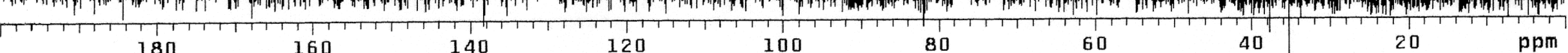


STANDARD IH OBSERVE

Pulse Sequence: s2pu

Solvent: $\operatorname{CDC} 13$

Temp. $25.0 \mathrm{C} / 298.1$

Pulse 90.0 degrees

Acq. time $3.744 \mathrm{sec}$

16 repetitions

OBSERVE H1, 299.9436011 MHZ

DATA PROCESSING
FT 5 ize 32768

Total time $1 \mathrm{~min}$, $0 \mathrm{sec}$
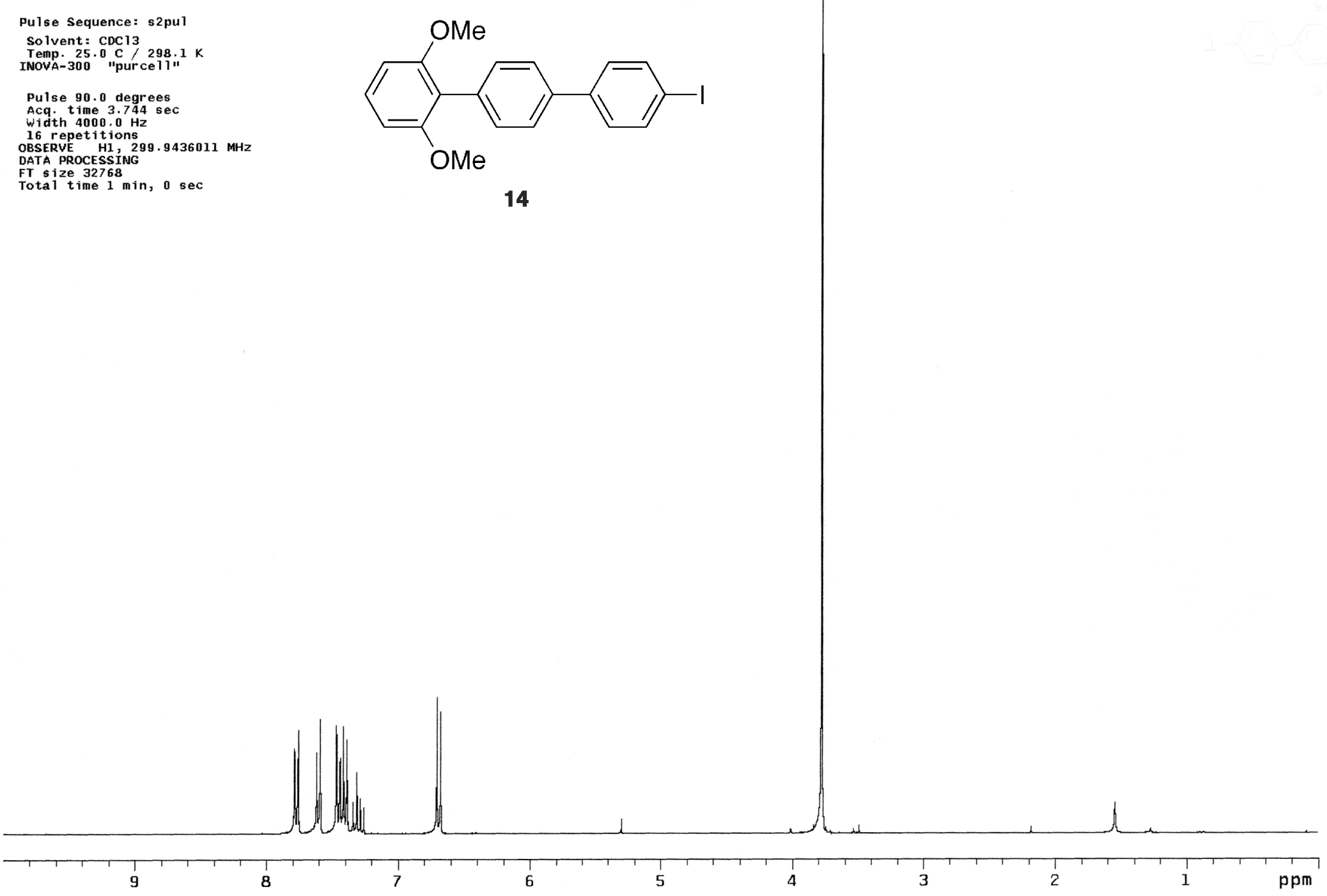
I3C OBSERVE

Pulse Sequence: s2pul Solvent: $\mathrm{CDCl}$ Temp. $25.0 \mathrm{C} / 298.1 \mathrm{~K}$
INOVA-300 "purce11"

Relax. delay $1.000 \mathrm{sec}$

Acq. $t$ ime 0.910 sec

OESERVE Cl3, 75.4208786

DEOULE H1, 299.9450944 MHz

cont inuous ly on

WALTZ-16 modulated

Line broadening $2.5 \mathrm{~Hz}$

Fotal time $5 \mathrm{hr}, 19 \mathrm{~min}, 49 \mathrm{sec}$
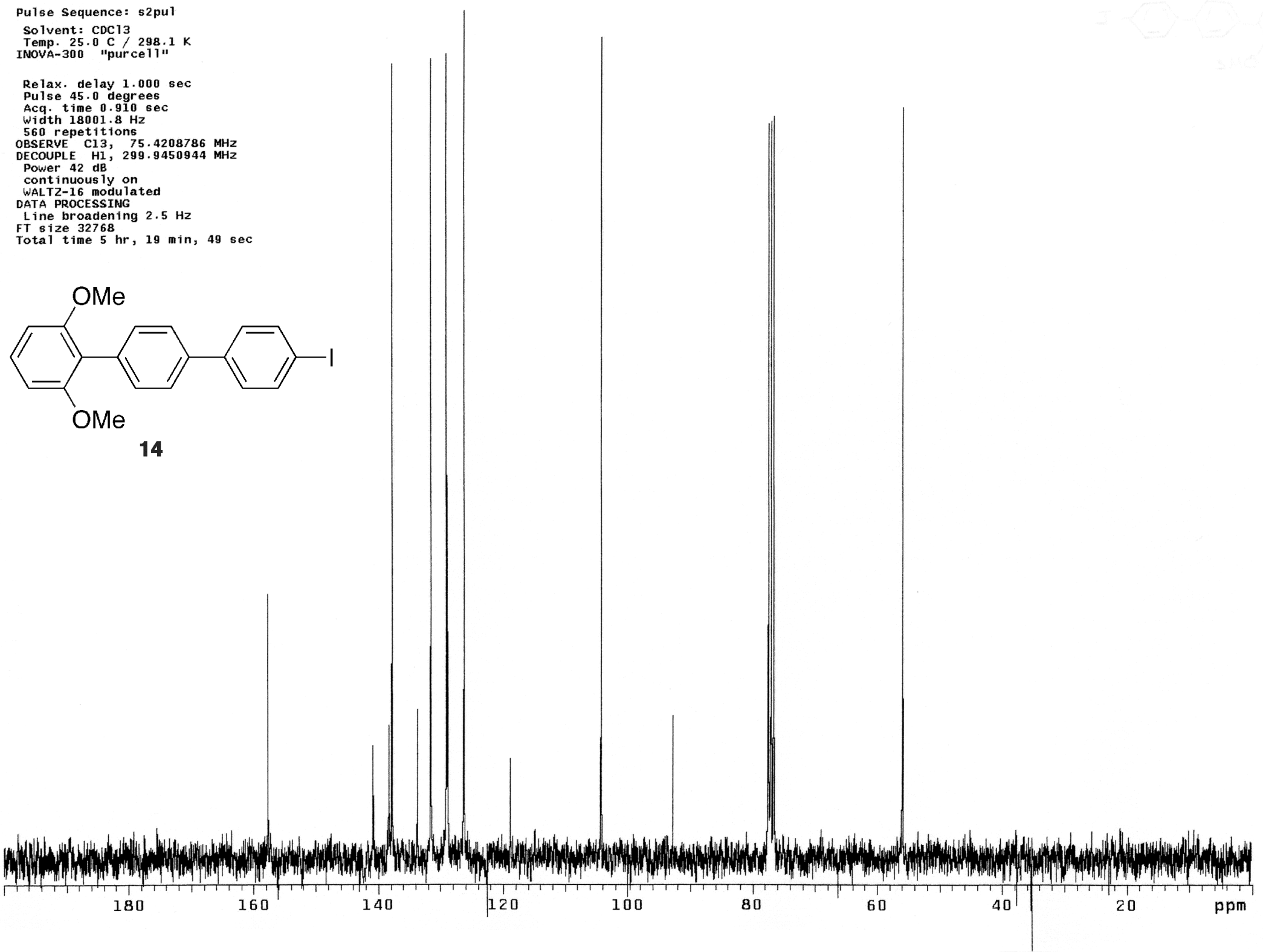
STANDARD IH OBSERVE

Pulse Sequence: s2pu

Solvent: $\operatorname{CDC13}$
Temp. $25.0 \mathrm{C} / 298.1 \mathrm{~K}$
INOVA-300 "purcel1"

INOVA-300 "purcell"

Pulse 90.0 degrees

Acq. time 3.744
width $4000.0 \mathrm{~Hz}$

16 repet $11,299.9436009 \mathrm{MHZ}$

DATA PROCESSING

FT size 32768
Total time $1 \mathrm{~min}, 0 \mathrm{sec}$

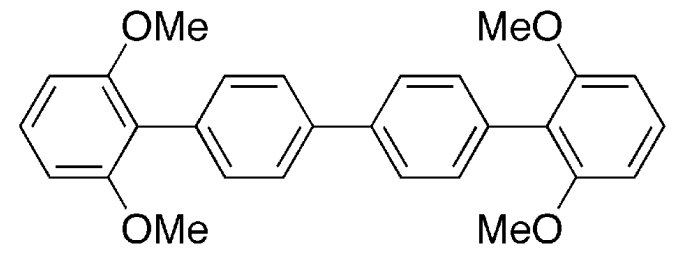

15

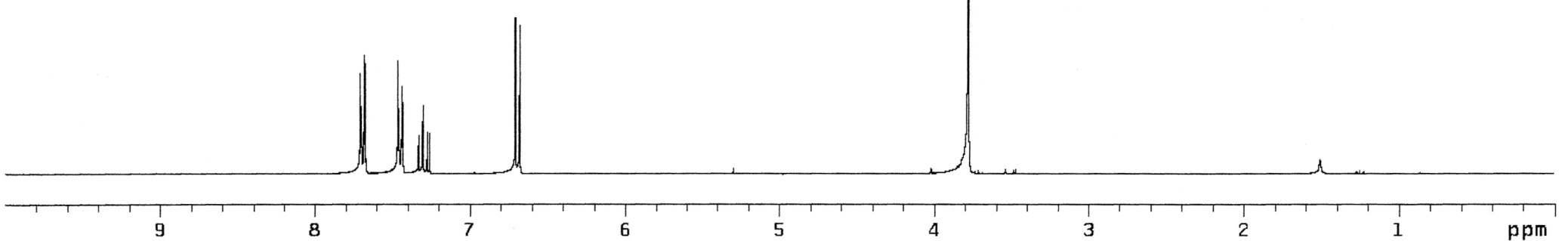


13C OBSERVE

Pulse Sequence: s2pul

Solvent: $\mathrm{CDCl} 13$
Temp. $25.0 \mathrm{C} / 298.1 \mathrm{~K}$
INOVA-300 "purce11"

Relax. delay $1.000 \mathrm{sec}$

Pcq. time $0.910 \mathrm{sec}$

Width $18001.8 \mathrm{H}$

OBSERE C13, 75.4208764 MH

cont inuous ly on
WALTZ-16 modulate

DATA PROCESSING

FT size 32768

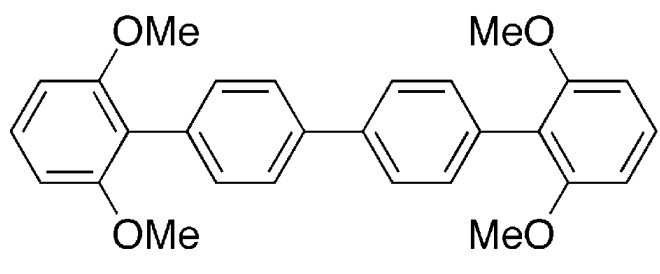

15
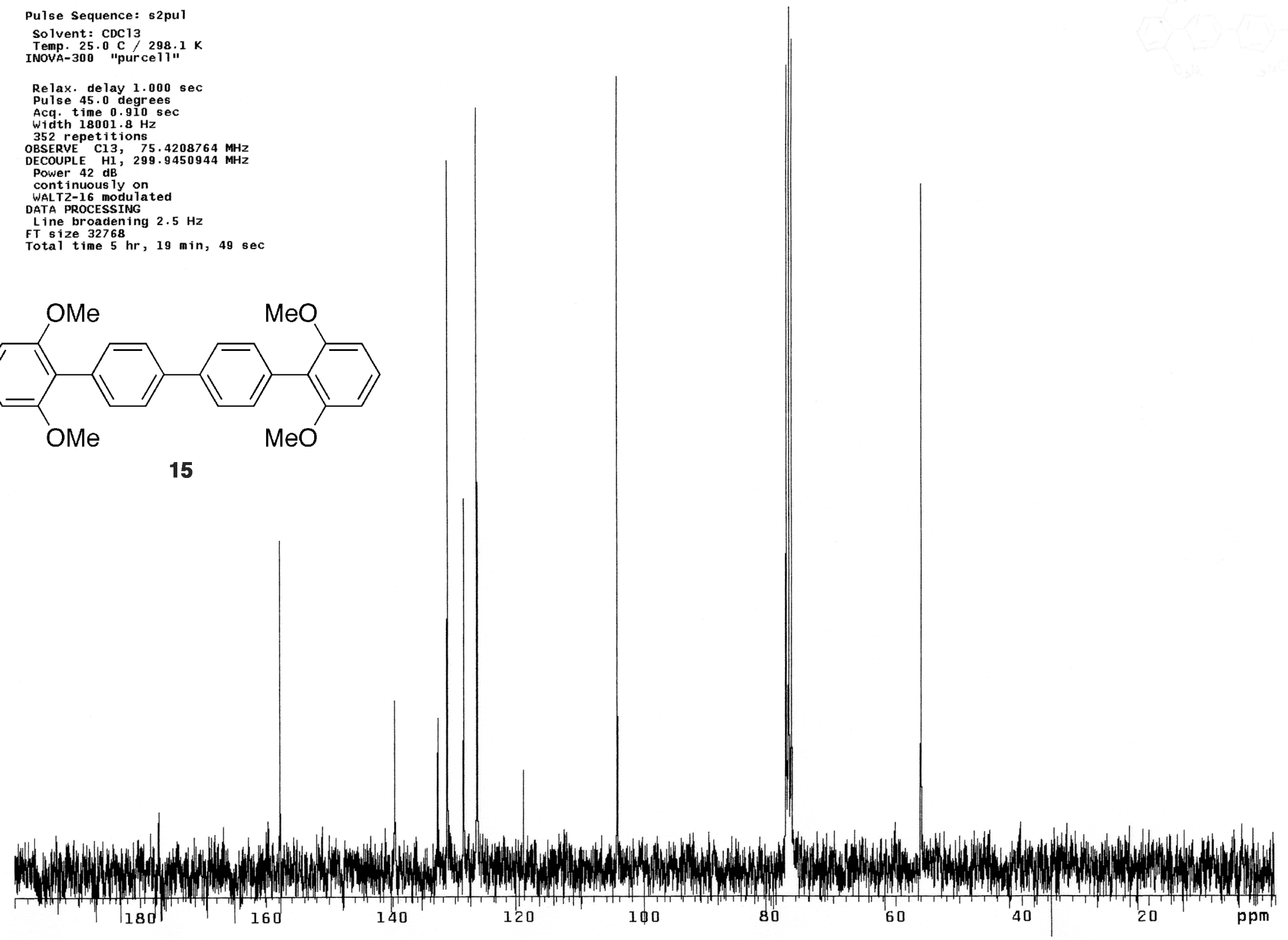
STANDARD IH OBSERVE

Pulse Sequence: s2pul

Solvent: $\mathrm{CDCl} \mathrm{K}$
Temp. $25.0 \mathrm{C} / 298.1 \mathrm{~K}$
INOVA-300 "purcel11"

Pulse 90.0 degrees

Acq. time 3.744 sec

16 repetitions

OBATRVE H1, $299.9436006 \mathrm{MHZ}$

DATA PROCESSING

Total time $1 \mathrm{~min}, 0 \mathrm{sec}$

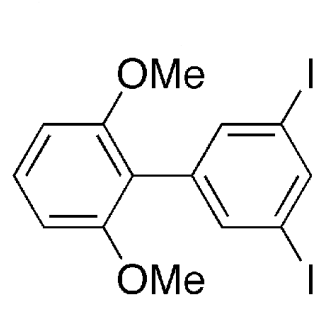

16

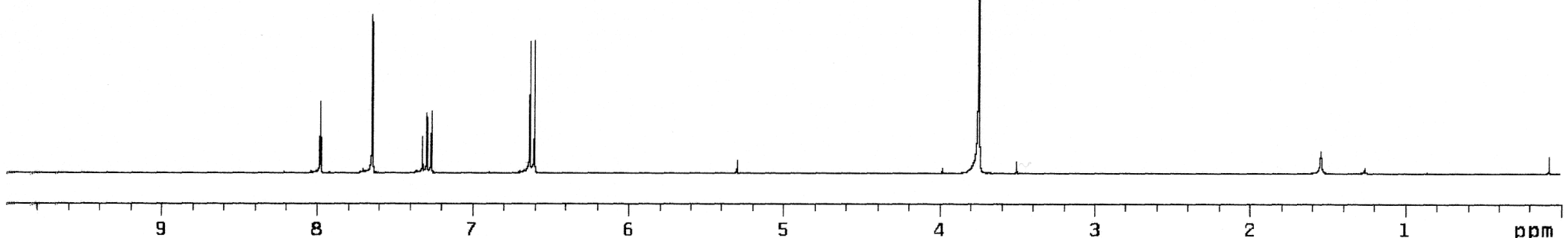


13C OBSERVE

Pulse Sequence: s2pul

Solvent: $\mathrm{CDCl}$

Temp. $25.0 \mathrm{C} / 298.1 \mathrm{~K}$

Relax. de lay $1.000 \mathrm{sec}$

Pulse 45.0 degrees

Acq. time $0.910 \mathrm{sec}$

656 repetition

OBSERVE Cl3, $75.4208764 \mathrm{MHZ}$

Power $42 \mathrm{~dB}$

cont inuous ly on

DATA PROCESSING

Line broadening $2.5 \mathrm{~Hz}$

FT size 32768
Total time $5 \mathrm{hr}, 19 \mathrm{~min}, 49 \mathrm{sec}$

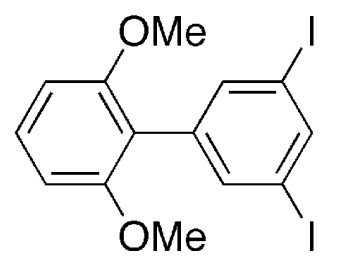

16

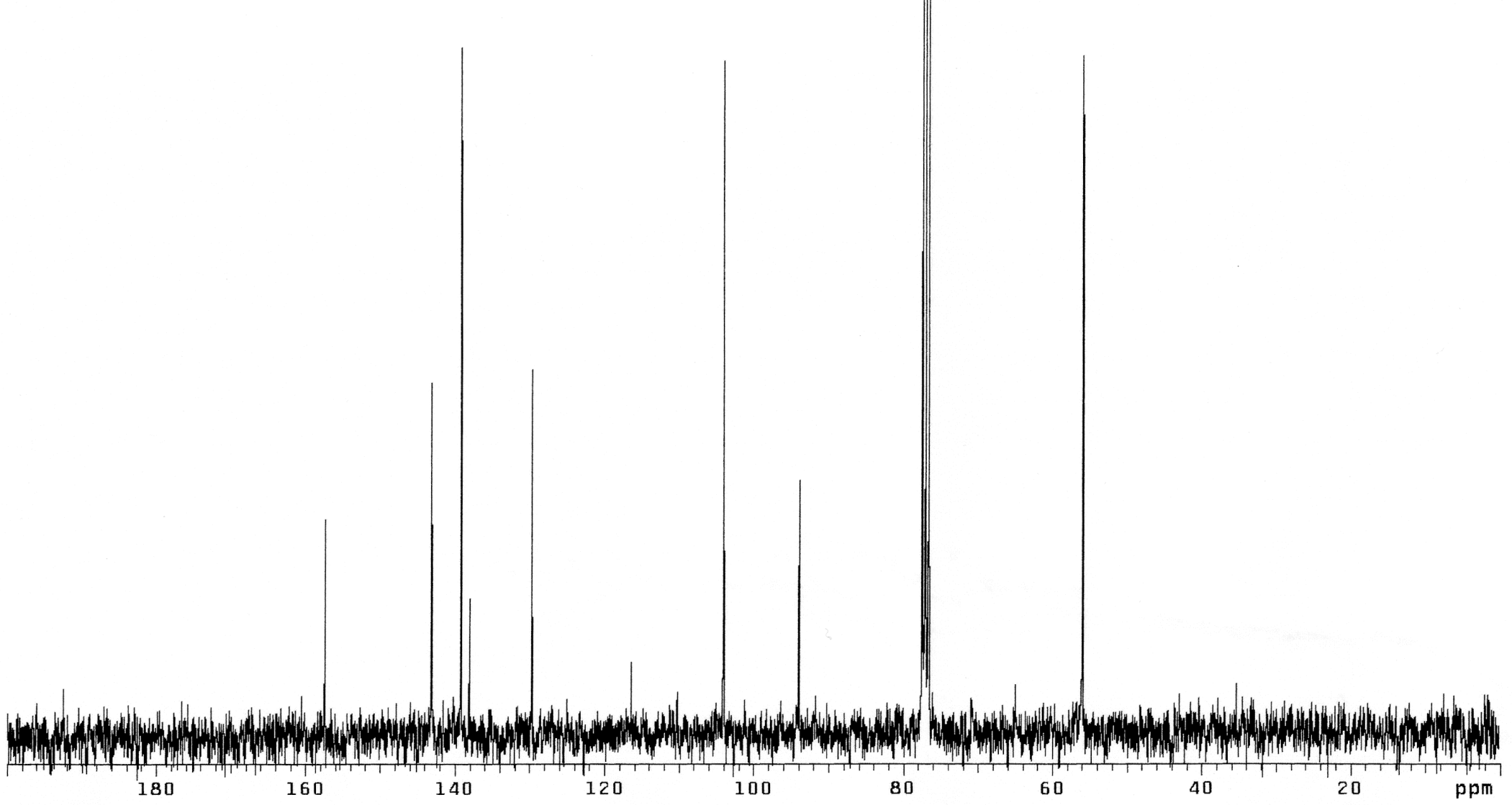


STANDARD IH OBSERVE

Pulse Sequence: s2pu

Solvent: $\operatorname{CDC} 13$
Temp. $25.0 \mathrm{C} / 298.1 \mathrm{~K}$
INOVA-300 "purcelli"

Pulse 90.0 degrees
Acr. time $3.744 \mathrm{sec}$

Acq. time 3.744
Width $4000.0 \mathrm{~Hz}$

16 repetitions

OATA PROCESSING 299.9436009 MHZ

FT size 32768
rotal time $1 \mathrm{~min}, 0 \mathrm{sec}$

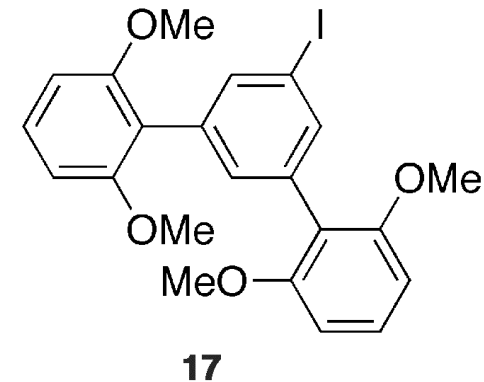

17

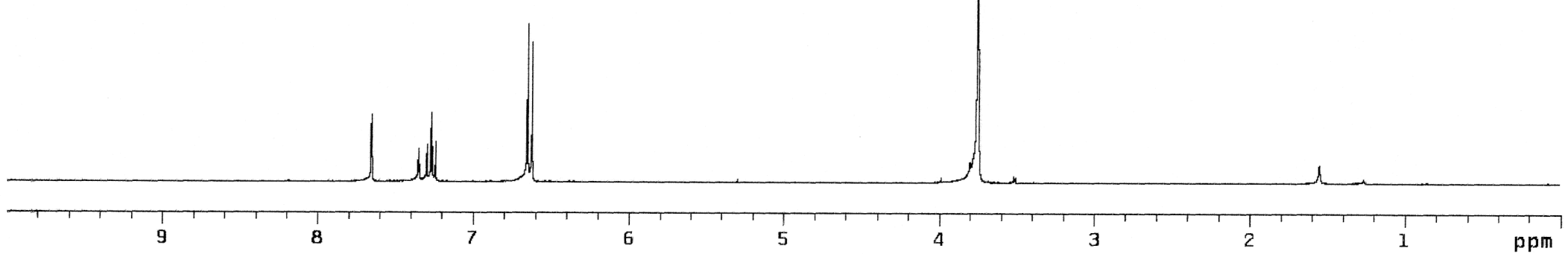


I3C OBSERVE

Pulse Sequence: s2pul

Solvent: CDC13
Temp. $25.0 \mathrm{C} / 298.1 \mathrm{~K}$
INOVA-300 "purcel1"

Relax. delay $1.000 \mathrm{sec}$

Pulse 45.0 degrees
Acq. $t$ ime $0.910 \mathrm{sec}$

width $18001.8 \mathrm{~Hz}$

OBSERVE C13, $75.4208775 \mathrm{MHZ}$

Power $42 \mathrm{~dB}$

Cont inuous ly on

Line broadening $2.5 \mathrm{~Hz}$

FT 5 jize 32768
Total time $5 \mathrm{hr}, 19 \mathrm{~min}, 49 \mathrm{sec}$

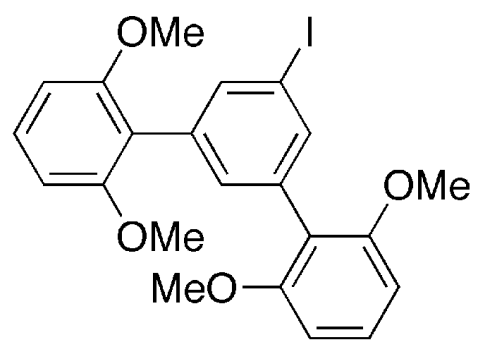

17

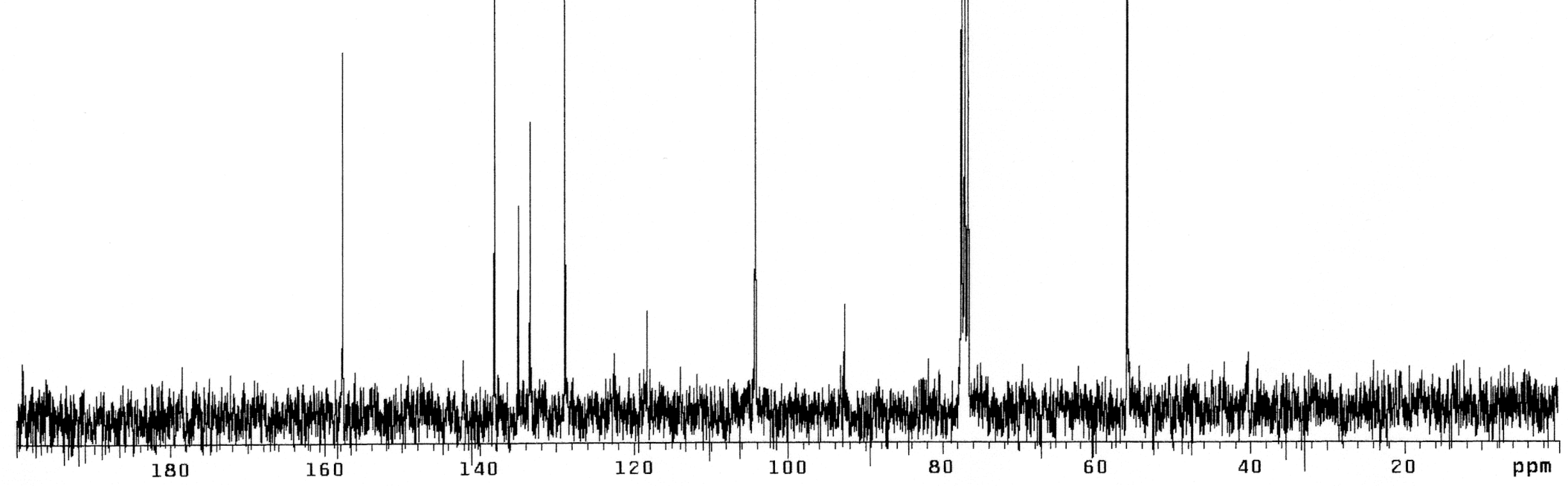


STANDARD IH OBSERVE

Pulse Sequence: s2pu

Solvent: $\operatorname{cDCl} 13$

Temp. $25.0 \mathrm{C} / 298.1 \mathrm{~K}$
INOVA-300 "purcel11"

Pu1se 90.0 degrees

Width $4000.0 \mathrm{~Hz}$

16 repetitions

DATA PROCESSING

FT size 32768
Total time $1 \mathrm{~min}, 0 \mathrm{sec}$

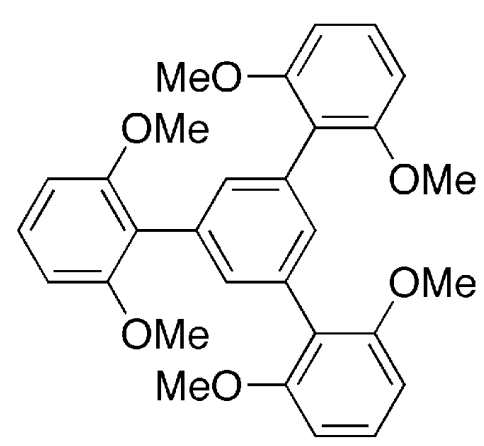

18

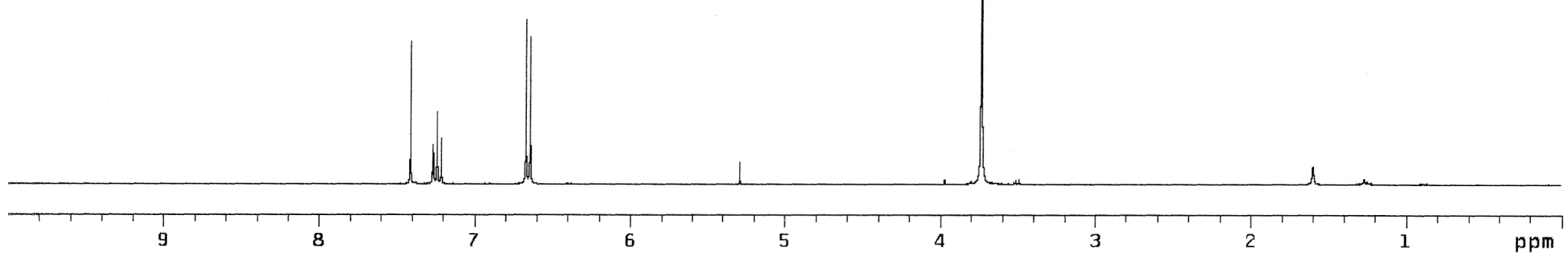


13C OBSERVE

Pulse Sequence: s2pur

Solvent: CDC13
Temp. $25.0 \mathrm{C} / 298.1 \mathrm{~K}$
INOVA-300 "purce11"

Relax. delay $1.000 \mathrm{sec}$

Pulse 45.0 degrees

Width $18001.8 \mathrm{~Hz}$

OBSERVE C13, $75.4208775 \mathrm{MHZ}$
DECOUPLE H1, 299.9450944 MHZ

Power $42 \mathrm{~dB}$

Cont inuous ly on
WALT $2-16$ modulated

DATA PROCESSING $2.5 \mathrm{~Hz}$

FT size 32768 ,

Total time $5 \mathrm{hr}, 19 \mathrm{~min}, 49 \mathrm{sec}$

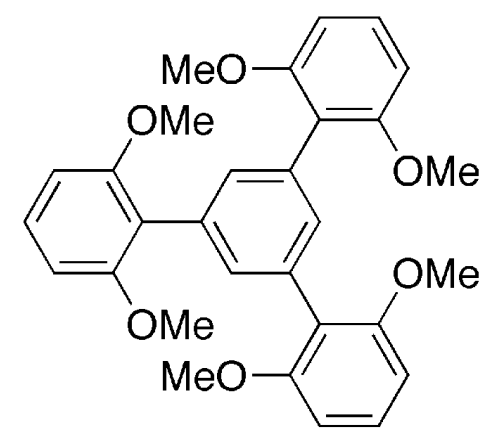

18

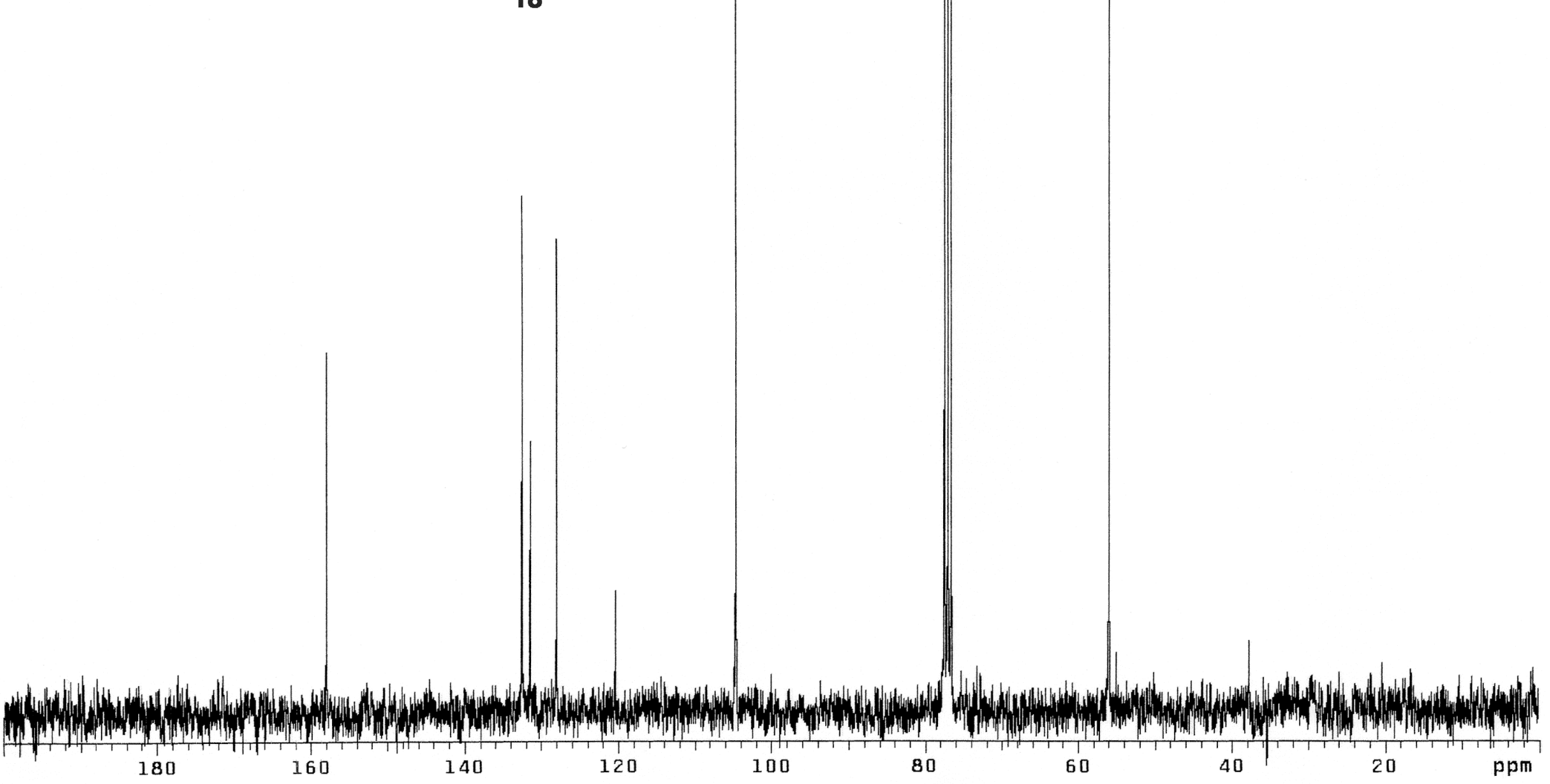

